\title{
Are early maladaptive schemas, temperament and character dimensions correlated?
}

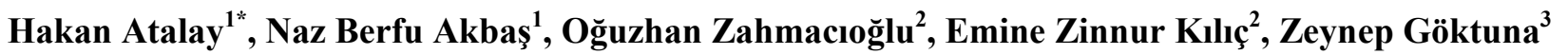 \\ ${ }^{1}$ Department of Psychiatry, Yeditepe University Medical School, Istanbul, Turkey \\ ${ }^{2}$ Department of Child and Adolescent Psychiatry, Yeditepe University Medical School, Istanbul, Turkey \\ ${ }^{3}$ Yeditepe University Medical School, Istanbul, Turkey \\ Email: "hakanatalay2005@gmail.com
}

Received 23 November 2012; revised 25 December 2012; accepted 3 January 2013

Copyright (C) 2013 Hakan Atalay et al. This is an open access article distributed under the Creative Commons Attribution License, which permits unrestricted use, distribution, and reproduction in any medium, provided the original work is properly cited.

\begin{abstract}
Objective: Aim of the study is to examine the relationships between early maladaptive schemas and the character and temperament aspects of personality, which are known as two approaches to the understanding of personality structure. Methods: We conducted the study using the Young Schema Questionnaire-Short Form (YSQ-SF) and the Cloninger's Temperament and Character Inventory (TCI) in 122 university students from different schools. Results and Conclusion: Results demonstrated that there are high level of positive as well as negative correlations between most of TCI items and some of the sub-scales of the YSQ, including vulnerability, social isolation and defectiveness. In consistent with the Halvorsen et al.'s findings, our study demonstrated that harm avoidance had a positive relationship with several EMSs, including emotional deprivation, deficiency, dependence, failure, subjugation and self-sacrifice. More importantly, we obtained the results consistent with theirs regarding the negative correlations between self-directedness and almost all of EMSs, with the exception of the schema of subjugation. We concluded that although some of the personality characteristics contained in the YSQ and the TCI are appeared to be related to each other, we need more studies to support the Young et al.'s suggestion that early maladaptive schemas (EMSs) are likely to reflect underlying characterological factors of personality.
\end{abstract}

Keywords: Early Maladaptive Schemas; Temperament; Character; Personality Development

\section{INTRODUCTION}

Psychodynamic approaches to personality development

"Corresponding author. prevailed the general opinion on the psychopathology and the treatment of psychiatric disorders for decades. In recent years there is, however, an increasing awareness of the formative effects by early life events among different psychological schools, including cognitive theory, or schema theory. Research in neuroscience immersed in such issues as attachment, early life traumas, etc. Converging evidence from such many areas of study appears to make the integration of these different views possible. Although psychodynamic explanations of personal development are increasingly replaced by two approaches to various aspects of personality, namely schema theory and cognitive theory, studies simultaneously dealt with both of these are scarce. In fact, common grounds may quite easily be viewed when these two approaches are simultaneously assessed. As prevailing view about causes and treatment of various psychopathologies, cognitive theory, for instance, asserts that the essence of personality is revealed in the dysfunctional beliefs that characterize and perpetuate it [1]. A second but similar theoretical approach has been adopted by Young [2]. Young has pointed out that a child's enduring experience of not getting his or her basic emotional needs in relationships with significant other will give rise to the development of early maladaptive schemas (EMSs). These schemas are defined as "extremely stable and enduring themes, comprised of memories, emotions, cognitions, and bodily sensations, regarding oneself and one's relationship with others, that develop during childhood and are elaborated upon throughout the individual's lifetime, and that are dysfunctional to a significant degree" [3]. From the first, cognitive point of view, Cloninger et al. initially postulated some temperamental aspects of personality, with all of them supposedly having psychobiological support [4-6]. They suggested that each of the temperaments is relatively stable and moderately heritable throughout life regardless of culture and social lear- 
ning [6]. They also outlined four dimensions of temperament as follows: Novelty seeking (NS) reinforces active approach behaviors with respect to novelty. Harm avoidance (HA) is associated with the avoidance of aversive stimuli. Reward dependence (RD) is related to the tendency to maintain behaviors in familiar situations or when rewards are expected. Persistence (P) leads to maintaining a behavior whatever its consequences [7]. Cloninger later realized that a description of temperament traits alone was not sufficient to distinguish patients from healthy controls and supplemented his theory of biogenetic temperament with the concepts of acquired characters [8]. Character dimensions mature in adulthood and influence personal and social effectiveness by learning about self-concepts. It involves higher cognitive processes and relatively conscious and explicit mechanisms than temperaments [9]. There are three character dimensions: Self-directedness (SD) refers to self-acceptance and responsibility. Cooperativeness $(\mathrm{C})$ refers to the ability to identify with and accept other people. Self-transcendence (ST) describes the tendency to feel part of nature and the universe [7,9-11]. All seven dimensions can be assessed using a self-report instrument, the Temperament Character Inventory (TCI) [6].

Some authors (e.g., Halvorsen et al.) claim that Cloninger et al.'s psychobiological model of personality is more comprehensive and elaborate in comparison to Young et al.'s schema theory [12]. However, the way temperament may interact with social experience in the development of schemas has so far received less attention [12]. Young et al. [3] in fact suggested that temperament plays an important role in interaction with adverse experience in childhood in the development of the EMSs although the schema theory does not describe how this interaction takes place. On the other hand, in Cloninger et al.'s model, the mechanisms of how the environment shapes the development of character traits are not further mentioned [12]. Therefore, it may be proposed that to the extent that temperamental dispositions influence actual and perceived encounters with the world, temperament would be expected to play an important role in the development of experiential schemas [13]. In other words, in the formation of schemas, innate temperament may have interacted with early adverse relational experiences [3]. So, the child's temperament plays a major role in the development of schemas since an extreme temperament makes the child more likely to be exposed to aversive parental rearing or may even override an ordinary early environment [14]. It means that the two different approaches can complement each other in contributing to a better understanding of psychological development of human being while there is some concern about possible overlap between EMSs, temperament and character traits. In this context, it may be useful to reflect upon Lohr et al.'s [13] explanation that there is a complementary relationship between EMSs and temperament. They argue that all of the schemas are mental structures that organize knowledge and facilitate problem solving thereby contributing to adjustment. Dysregulation in the cognitive, attentional and emotional systems of temperament may adversely influence the actual transactions between the individual and the environment, and may disrupt the organization of experiences into useful schematic structures. For example, a sense of not being in control of one's inner states due to temperamental reactivity or difficulty regulating attention may translate into schematic representations of lack of control. From this point of view temperament and character traits are expected to influence the child's interactions and the synthesis of accumulating experiences into schematic frameworks that inform the individual's subsequent encounters and constitute a storehouse of adaptive resources with implications for development. Assuming that risk or protective factors exert their influence on development by shaping the individual's transactions with the environment, then, both temperament and schemas serve these functions [13]. In conclusion, from a more theoretical point of view, the examination of the relationships between EMSs, the temperament and character dimensions are important because temperament is considered a significant vulnerability factor for the formation of EMSs [14]. Although it is most likely to have mutual relationships between the schemas, and the temperament and character domains, and it might be very significant clinically as well as theoretically to demonstrate the validity of these assumptions, it is obvious that to conduct such an investigation appears almost impracticable in many respects. For those obvious reasons, in the present study we aimed only to examine only statistically the relationships between early maladaptive schemas and the temperament and character dimensions, without any presupposition about these implications, using the Young Schema Questionnaire (YSQ) and the Cloninger's Temperament and Character Inventory in a non-clinical population of university students.

\section{MATERIALS AND METHODS}

\subsection{Participants}

The study sample consisted of the students from different schools at the Yeditepe University. After the study was approved by the Ethics Committee for Scientific Research of the University, the subjects were randomly selected for practical reasons according to the assumption that the student groups should be from different schools of the university so that they are likely to come from different backgrounds and thus to represent different characteristics. The aim, therefore, was primarily to form a convenience sample of subjects from a variety of 
faculties such as medical, psychology, fine arts, and dentistry and prep classes. Selected thus randomly the sample consisted of total 122 students from the psychology (n: 18), fine arts (n: 33), medical (n: 26), dentistry (n: 14$)$ and prep schools (n: 31 ), who were $63 \%$ of the students available at the classes at the time of the study. The data of the rest (37\%) were excluded because of various reasons such as unwillingness to participate, deficient information, etc. After giving detailed explanations about the study objectives, the psychology students who were actively taken part in any academic research at least once before administered the measures to the participants and obtained their written informed consents.

\subsection{Instruments}

The Temperament and Character Inventory (TCI): The TCI is a 240 -item self-report inventory with a true/false response format designed to assess individual differences on the basic dimensions of temperament and character [4]. It measures the four higher order temperament dimensions novelty seeking, harm avoidance, reward dependence and persistence, and the three higher order character dimensions self-directedness, cooperativeness and self-transcendence by related sub-scales of each dimension. The TCI was found to be an internally consistent and factor-analytically valid instrument $[9,10,14-23]$. The reliability and validity of the Turkish version of the TCI were supported by its reliable psychometric properties and construct validity in the Köse et al.'s study [19].

The Young Schema Questionnaire-Short Form (YSQSF): The original version of Schema Questionnaire was developed by Young to measure early maladaptive schemas, and it was a 205 -item self-report measure designed to assess most of the 18 schemas [2]. Research has provided support for the psychometric properties of the YSQ [24-27]. The Schema Questionnaire-Short Form (YSQ-SF) was also designed [28-31] to measure 15 maladaptive schemas and it is a briefer (75-item) instrument. Relative to the original 205-item version, the 75 -item version of Young Schema Questionnaire clearly has practical advantages for the clinician or researcher who wishes to investigate the core beliefs of individuals with psychological disorders [30]. Each item reflects a thought, feeling, or behavior that corresponds to a particular schema. Participants are instructed to rate each item on a Likert scale ranging from 1 to 6 , where 1 is "completely untrue of me" and 6 "describes me perfectly". It was found that YSQ-SF has high internal consistency and that it may be used with confidence in place of the more unwieldy 205-item version [24-27]. Soygüt et al.'s findings showed that the factor structure of the Turkish version of the YSQ-SF was generally consistent with previous studies and that it had acceptable levels of reliability and validity [32]. In addition, Pearson coeffi- cients for sub-scales varied between $r=0.66$ and $r=0.82$ $(\mathrm{p}<0.01)$. According to the test-retest reliability analysis, Pearson's correlation coefficients for schema domains varied between $r=0.66$ and $r=0.83(p<0.01)$. These results show that the coefficients obtained are significant and in the acceptable range. The internal consistency coefficient for the YSQ-SF3 sub-scales varied between $a$ $=0.53$ and $a=0.81$. Thus it is possible to say that the scale has a medium level of internal consistency.

\subsection{Statistical Methods}

Data was analyzed using the SPSS 16.0 package. Independent t-test was used for comparisons between continuous variables while $\chi^{2}$ analyses were used for categoric variables. Significance was accepted as $\mathrm{p}<0.05$.

\section{RESULTS}

The study sample consisted of 122 students ( 78 female, 44 male). Their mean age was 22.3 (ranging from 18 to $35 ; 22.5$ for female and 21.9 for male students), with no significant difference between them ( $\mathrm{t}: 0.97, \mathrm{p}>0.05)$. Table 1 illustrates the complex relationships between early maladaptive schemas and temperament and character dimensions. The comparisons of the YSQ subscales with the TCI items individually suggest that there are positive as well negative correlations between the scores from both of the measures. Close relationships were observed between most of TCI items and several sub-scales of the YSQ including vulnerability, social isolation, and defectiveness. Overall, the TCI had positive correlations with the YSQ sub-scales on items such as anticipatory worry $(0.44)$, shyness $(0.34)$, fatigability $(0.30)$, self-forgetfulness $(0.26)$ while statistically significant negative correlations were found in most of the items including explaratory activity, dependence, responsibility, purposefulness, resourcefulness, self-acceptance, congruent second nature, social acceptance, empathy, helpfulness, and compassion.

Table 1 reflects the relationships between the TCI items and the subscales of the YSQ as Pearson correlation coefficients $(r<0.05)$. Colored in order to demonstrate correlations more clearly, Table 2 shows that the character dimension self-directedness has the highest level of (negative) correlation with the YSQ sub-scales. The TCI items individually demonstrate strongly negative correlations with most of the YSQ domains, especially with those of disconnection/rejection and impaired autonomy/performance.

Table 3 more clearly illustrates these relationships in comparing the main sub-dimensions of the TCI with all items of the EMSs. As it can be seen in Table 3, selfdirectedness as a whole was negatively correlated with all sub-scales of the YSQ only except for subjugation. 


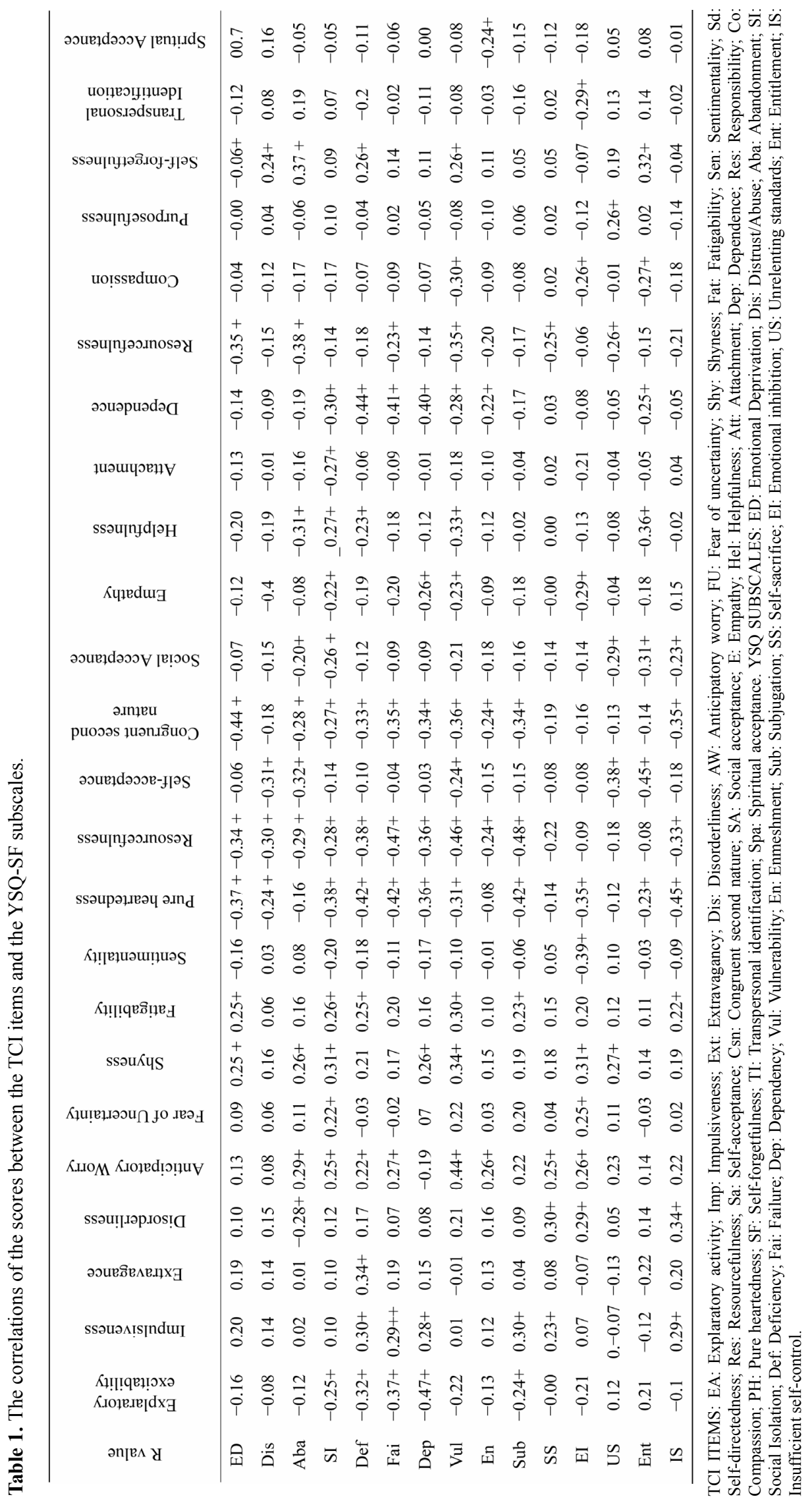


Table 2. The correlations of the scores are shown below in a colored table in order to illustrate more clearly the relationships between the TCI and the YSQ-SF where red cells represent statistically significant negative correlations while blue cells represent statistically significant positive correlations.

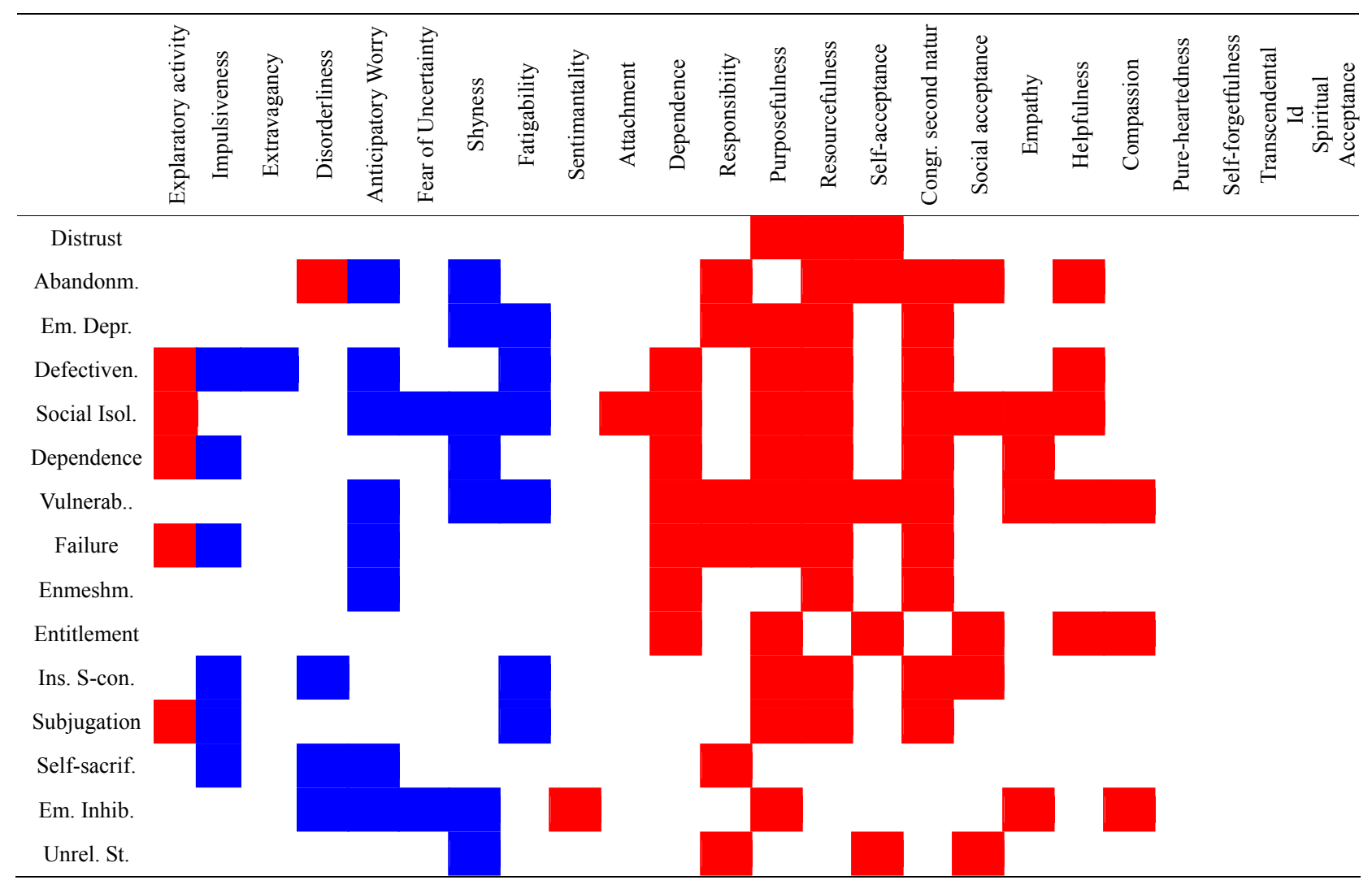

Table 3. Relationships between the temperament and character dimensions of the TCI and the subscales of the YSQ-SF as shown in the significant correlations between them. (NS: Novelty seeking; HA: Harm-avoidance; RD: Reward dependence; P: Persistence; SD: Self-directedness; C: Cooperativeness; ST: Self-transcendence).

\begin{tabular}{|c|c|c|c|c|c|c|c|}
\hline & NS & HA & $\mathrm{RD}$ & $\mathrm{P}$ & S-D & $\mathrm{C}$ & S-T \\
\hline Distrust & 0.07 & 0.25 & -0.24 & -0.12 & $-0.44+$ & -0.12 & 0.01 \\
\hline Abandonment & 0.00 & 0.09 & -0.06 & $-0.29+$ & $-0.34+$ & -0.10 & $0.23+$ \\
\hline Em. Depriv. & -0.02 & $0.28+$ & $-0.23+$ & $-0.24+$ & $-0.45+$ & -0.21 & $-0.23+$ \\
\hline Defectiveness & -0.10 & $0.32+$ & $-0.38+$ & -0.06 & $-0.26+$ & -0.20 & 0.01 \\
\hline Social Isolation & 0.05 & 0.20 & $-0.39+$ & -0.03 & $-0.39+$ & -0.11 & 0.11 \\
\hline Dependence & 0.05 & $0.22+$ & $-0.29+$ & $-0.29+$ & $-0.41+$ & -0.12 & 0.04 \\
\hline Vulnerability & -0.02 & 0.21 & $-0.28+$ & $-0.24+$ & $-0.30+$ & -0.12 & 0.03 \\
\hline Failure & -0.01 & $0.43+$ & $-0.29+$ & -0.06 & $-0.51+$ & $-0.29+$ & 0.04 \\
\hline Enmeshment & 0.07 & 0.19 & 0.09 & 0.03 & $-0.25+$ & -0.11 & -0.08 \\
\hline Entitlement & 0.03 & 00.26 & -0.18 & $-0.27+$ & $-0.44+$ & -0.09 & -0.12 \\
\hline Insufficient $\mathrm{SCl}$ & $0.25+$ & 0.19 & 0.05 & $-0.29+$ & $-0.29+$ & -0.00 & -0.03 \\
\hline Subjugation & -0.05 & $0.32+$ & $-0.36+$ & -0.14 & -0.16 & -0.21 & -0.21 \\
\hline Self-sacrifice & 0.01 & $0.26+$ & 0.04 & 0.17 & $-0.34+$ & -0.02 & 0.17 \\
\hline Em. Inh. & 0.19 & 0.12 & -0.15 & -0.06 & $-0.30+$ & $-0.34+$ & $0.27+$ \\
\hline Unrele. Stand. & $0.31+$ & 0.19 & -0.03 & $-0.32+$ & $-0.42+$ & -0.13 & 0.03 \\
\hline
\end{tabular}


Persistence and reward dependence also demonstrated negative correlations, though less, with most of the YSQ sub-scales. On the contrary, there was a positive correlation between harm avoidance and YSQ items suggesting that these domains are likely to show similar characteristics of personality.

\section{DISCUSSION}

The present study sought to show the relationships between the YSQ-SF scales and the dimensions of the Character and Temperament Inventory. Although researches have largely demonstrated that the early maladaptive schemas were correlated with a wide spectrum of psychopathology and personality disorders in general, and with specific personality disorder traits in particular [33], there was scarcity of studies investigating simultaneously the relationships between EMSs, and the temperament and character traits. Thus, by relating the YSQ-SF scales to the temperament and character traits of the TCI, the study may have a modest contribution to the understanding of the EMSs and associated temperamental and character tendencies. Although causal inferences can not be made due to many factors inherent in the study including the cross-sectional design and small sample size, our findings seem to be consistent in some respects with recent research as well as with Young et al.'s schema model that asserts a relationship between innate temperament and EMSs. In their studies, for example, on early maladaptive schemas, temperament and character traits in clinically depressed and previously depressed subjects, Halvorsen et al. [12] reported positive correlations of EMSs with harm avoidance and negative associations with self-directedness, persistence, and cooperativeness. In consistent with their findings, our study demonstrated that harm avoidance had a positive relationship with several EMSs, including emotional deprivation, deficiency, dependence, failure, subjugation, and self-sacrifice. In this context, Halvorsen et al.'s explanation that this result may be due to the fact that the development of such EMSs in early adverse relationship patterns interacts with a temperament of being anxious, pessimistic and shy, as reflected by high harm avoidance, is applicable to our study. More importantly, we obtained the results consistent with those of the Halvorsen et al.'s regarding the negative correlations between self-directedness and almost all of EMSs, with the exception of the schema of subjugation. This is important because the character trait self-directedness reflects a mature selfconcept while EMSs reflect negative core beliefs regarding self and personal relationships [12]. These overlaps between our study results and those of Halvorsen et al.'s support the Young et al.'s suggestion that EMSs are reflecting underlying characterological factors.
Lack of correlation between EMSs and the temperament novelty seeking and the characters cooperativeness and self-transcendence are worth thinking because this may make the relationships of early maladaptive schemas, which are originated in early adverse relationship patterns, and temperament and character dimensions, which are largely thought to be hereditary, disputable in some respects. However, we should keep in mind that, according to Young et al., a vulnerable temperament is not sufficient for the formation of EMSs. More important are the ways in which the early environment handles the child's temperament. Future studies, therefore, should focus on the interaction between temperament and adverse relational experiences when investigating the development of EMSs.

Overall, there seems to be an increase in research aimed to better understand personality development since early years of life and the progresses in this area appear to make valuable contributions to a variety of psychotherapies, including cognitive and dynamic ones. Thus, tendency to integrate all of these approaches to human mind and behavior is expected more likely to strengthen in time, and research on the impact of the relationships with early significant others and life events on the formation of the character and the role of early maladaptive schemas in this processes will give significant cues for this integration attempts.

So, there may be an asset of the present study because of being conducted in a non-clinical population, but it suffered from various limitations, too. First, it should be borne in mind that the study was cross-sectional in nature. Thus, no conclusions can be drawn about cause-effect relationship between maladaptive schemas, on one hand, and temperament and character on the other hand. Moreover, some of the TCI temperamental dimensions and the EMSs seem to have overlapping conceptual content, for instance, harm avoidance and vulnerability to harm. It may be led to some problems for analyses and interpretation of results, in particular in a cross-sectional study like this. Second, the study relied on a sample of students from a private/foundation college. It would be interesting to study more students from other colleges that are probably in more varied socioeconomic conditions than in our study sample. However, this, in fact, may not yield to a difference in terms of the study aims because our primary object was mainly to examine statistically the relations between the YSQ-SF and the dimensions of TCI. One more limitation of the study is its reliance on self-reports. EMSs are, by definition, partly unconscious. Thus, the YSQ-SF measures only EMSs of which an individual is aware. Moreover, coping responses to EMSs as schema avoidance may have influenced the completion of the inventory.

With keeping all in mind, it seems important for future 
research to use projective tests or physiological indicators of information processing in the assessment of EMSs. Also, although the present findings suggest that some impact of temperament and character on the development of EMSs may vary between different EMSs, long-term longitudinal studies are needed to answer the question of how adverse relational experiences interact with temperament/character in the development of EMS.

\section{REFERENCES}

[1] Beck, A.T., Brown, G., Dahlsgaard, K., Newman, C.F. and Beck, J.S. (2001) Dysfunctional beliefs discriminate personality disorders. Behaviour Research and Therapy, 39, 1213-1225. doi:10.1016/S0005-7967(00)00099-1

[2] Young, J.E. and Brown, G. (2001) Young schema questionaire. In: Cognitive Therapy for Personality Disorders, Professional Resource Press, Sarasota.

[3] Young, J.E., Klosko, J.S. and Weishaar, M.E. (2003) Schema therapy: A practioner's guide New York. Guilford Publications, New York.

[4] Cloninger, C.R., Svrakic, D.M. and Przybeck, T.R. (1993) A psychobiological model of temperament and character. Archives of General Psychiatry, 50, 975-990. doi:10.1001/archpsyc.1993.01820240059008

[5] Cloninger, C.R. (1994) Temperament and personality. Current Opinion in Neurobiology, 4, 266-273. doi:10.1016/0959-4388(94)90083-3

[6] Cloninger, C.R., Bayon, C. and Svrakic, D.M. (1998) Measurement of temperament and character in mood disorders: A model of fundamental states as personality types. Journal of Affective Disorders, 51, 21-32. doi:10.1016/S0165-0327(98)00153-0

[7] Matsudaira, T. and Kitamura, T. (2006) Personality traits as risk factors of depression and anxiety among Japanese students. Journal of Clinical Psychology, 62, 97-109. doi: $10.1002 /$ jclp. 20215

[8] Cloninger, C.R. (2004) Feeling good: The science of well-being. Oxford University Press, New York.

[9] Ha, J.H., Kim, E.J., Abbey, S.E. and Kim, T.-S. (2007) Relationship between personality disorder symptoms and temperament in the young male general population of South Korea. Psychiatry and Clinical Neurosciences, 61, 59-66. doi:10.1111/j.1440-1819.2007.01611.x

[10] Marteinsdottir, I., Tillfors, M., Furmark, T., Anderberg, U.M. and Ekselius, L. (2003) Personality dimensions measured by the Temperament and Character Inventory (TCI) in subjects with social phobia. Nordic Journal of Psychiatry, 57, 29-35. doi:10.1080/08039480310000239

[11] Paris, J. (2005) Neurobiological dimensional models of personality: A review of the models of Cloninger, Depue, and Siever. Journal of Personality Disorders, 19, 156170. doi:10.1521/pedi.19.2.156.62629

[12] Halvorsen, M., Wang, C.E., Richter, J., Myrland, I., Pedersen, S.K., Eisemann, M., et al. (2009) Early maladaptive schemas, temperament and character traits in clinically depressed and previously depressed subjects.
Clinical Psychology and Psychotherapy, 16, 394-407. doi:10.1002/cpp.618

[13] Lohr, L.B., Teglasi, H. and French, M. (2004) Schemas and temperament as risk factors for emotional disability. Personality and Individual Differences, 36, 1637-1654. doi:10.1016/j.paid.2003.06.011

[14] Thimm, J.C. (2010) Personality and early maladaptive schemas: A five-factor model perspective. Journal of Behavior Therapy and Experimental Psychiatry, 41, 373380. doi:10.1016/j.jbtep.2010.03.009

[15] Arkar, H., Sorias, O., Tunca, Z., Şafak, C., Alkin, T., Akdede, B., et al. (2005) Factorial structure, validity, and reliability of the Turkish temperament and character inventory. Turkish Journal of Psychiatry, 16, 190-204.

[16] Bayon, C., Hill, K., Svrakic, D.M., Przybeck, T.R. and Cloninger, C.R. (1996) Dimensional assessment of personality in an out-patient sample: relations of the systems of Millon and Cloninger. Journal of Psychiatric Research, 30, 341-352. doi:10.1016/0022-3956(96)00024-6

[17] Çelikel, F.C., Köse, S., Cumurcu, B.E., Erkorkmaz, U., Sayar, K., Borckardt, J., et al. (2009) Cloninger's temperament and character dimensions of personality in patients with major depressive disorder. Comprehensive Psychiatry, 50, 556-561. doi:10.1016/j.comppsych.2008.11.012

[18] Kim, S.J., Lee, J., Yune, S.K., Sung, Y.H., Bae, S.C., Chung, A., et al. (2006) The relationship between the biogenetic temperament and character and psychopathology in adolescents. Psychopathology, 39, 80-86. doi:10.1159/000090597

[19] Köse, S., Sayar, K., Kalelioğlu, U., Aydın, N., Çelikel, F.C., Güleç, H., et al. (2009) Normative data and factorial structure of the Turkish version of the Temperament and Character Inventory. Comprehensive Psychiatry, 50, 361368. doi:10.1016/j.comppsych.2008.09.007

[20] MacDonald, D.A. and Holland, D. (2002) Examination of relations between the neo personality inventory-revised and the temperament and character inventory. Psychological Report, 91, 921-930.

[21] Mulder, R.T., Joyce, P.R., Sullivan, P.F., Bulik, C.M. and Carter, F.A. (1999) The relationship among three models of personality psychopathology: DSM-III-R personality disorder, TCI scores and DSQ defences. Psychological Medicine, 29, 943-951. doi:10.1017/S0033291799008533

[22] Rie, S.M. de la, Duijsens, D.C. and Cloninger, C.R. (1998) Temperament, character, and personality disorders. Journal of Personality Disorders, 12, 362-372. doi:10.1521/pedi.1998.12.4.362

[23] Trouillet, R. and Gana, K. (2008) Age differences in temperament, character and depressive mood: A crosssectional study. Clinical Psychology and Psychotherapy, 15, 266-275. doi:10.1002/cpp.580

[24] Hoffart, A., Sexton, H., Hedley, L.M., Wang, C.E., Holthe, H. and Haugum, J.A. (2005) The structure of maladaptive schemas: A confirmatory factor analysis and a psychometric evaluation of factor-derived scales. Cognitive Therapy and Research, 29, 627-644. doi:10.1007/s10608-005-9630-0

[25] Muris, P. (2006) Maladaptive schemas in non-clinical 
adolescents: Relations to perceived parental rearing behaviours, big personality factors and psychopathological symptoms. Clinical Psychology and Psychotherapy, 13, 405-413. doi:10.1002/cpp.506

[26] Petrocelli, J.V., Glaser, B.A., Calhoun, G.B. and Campbell, L.F. (2001) Early maladaptive schemas of personality disorder subtypes. Journal of Personality Disorders, 15, 546-559. doi:10.1521/pedi.15.6.546.19189

[27] Schmidt, N.B., Joiner, T.E., Young, J.E. and Telch, M.J. (1995) The Schema-Questionnaire: Investigation of psychometric properties and the hierarchical structure of a measure of maladaptive schemas. Cognitive Therapy and Research, 19, 295-321. doi:10.1007/BF02230402

[28] Calvete, E., Estevez, A., Arroyabe, L.E. and Ruiz, P. (2005) The Schema Questionnaire-Short Form: Structure and relationship with automatic thoughts and symptoms of affective disorders. European Journal of Psychological Assessment, 21, 90-99. doi:10.1027/1015-5759.21.2.90

[29] Stopa, L., Thorne, P., Waters, A. and Preston, J.J. (2001) Are the short and long forms of the Young Schema Questionnaire comparable and how well does each version predict psychopathology scores? Journal of Cognitive Psychotherapy, 15, 253-272.

[30] Waller, G., Meyer, C. and Ohanian, V. (2001) Psychometric properties of the long and short versions of the Young Schema Questionnaire: Core beliefs among bulimic and comparison women. Cognitive Therapy and Research, 25, 137-147. doi:10.1023/A:1026487018110

[31] Welburn, K., Coristine, M., Dagg, P., Pontefract, A. and Jordan, S. (2002) The Schema Questionnaire-Short Form: Factor analysis and relationship between schemas and symptoms. Cognitive Therapy and Research, 26, 519-530. doi:10.1023/A:1016231902020

[32] Soygut, G., Karaosmanoğlu, A. and Çakır, Z. (2009) Assessment of early maladaptive schemas: A psychometric study of the Turkish Young Schema Questionnaire-Short Form-3. Turkish Journal of Psychiatry, 20, 75-84.

[33] Nordahl, H.M., Holthe, H. and Haugum, J.A. (2005) Early maladaptive schemas in patients with or without personality disorders: Does schema modification predict symptomatic relief? Clinical Psychology and Psychotherapy, 12, 142-149. doi:10.1002/cpp.430 